Technical Note

\title{
Numerical implementation of thermal boundary conditions in the lattice Boltzmann method
}

\author{
Long-Sheng Kuo, Ping-Hei Chen* \\ Department of Mechanical Engineering, National Taiwan University, No. 1, Sec. 4, Roosevelt Road, Taipei 10617, Taiwan, ROC
}

\section{A R T I C L E I N F O}

Article history:

Received 21 May 2008

Received in revised form 16 July 2008

Available online $\mathrm{xxxx}$

\section{Keywords:}

Lattice Boltzmann method

LBM

Rayleigh-Bénard convection

\begin{abstract}
A B S T R A C T
This work proposed a non-equilibrium mirror-reflection scheme to implement thermal boundary conditions for the two-distribution lattice Boltzmann method (TLBM). The study showed that the most popular non-equilibrium bounce-back scheme would become inadequate when the predictions of temperature gradient were examined in TLBM. This work used the native method in TLBM to verify temperature gradient instead of the conventional finite difference approximation. The simulation results demonstrated that the mirror-reflection scheme is a scheme of second-order accuracy and can predict the temperature and temperature gradient correctly. With help of calculating the heat flux on the boundary, this work also suggested a more efficient and realistic way to determine the Nusselt number in Rayleigh-Bénard convection problems.
\end{abstract}

(c) 2008 Elsevier Ltd. All rights reserved.

\section{Introduction}

Recently there are a lot of researches applying the two-distribution lattice Boltzmann method (TLBM) to study thermal systems [1-3]. They have demonstrated that TLBM is a powerful numerical tool in solving the temperature distributions. Very interestingly, however, to the authors' knowledge, there is no literature to discuss other important thermal properties like heat flux. Although basically heat flux can be determined from the temperature field using the conventional Fourier law, TLBM also has the capability to calculate the heat flux from the microscopic point of view. Thus, it is important to verify it from direct calculations in TLBM. On the other hand, the schemes to impose boundary conditions are important for numerical methods. The commonest scheme applied, if not overestimated, for boundary conditions in TLBM is the non-equilibrium bounce-back scheme [2-4]. It has shown successes in solving the temperature distributions. But when further studying the properties of heat flux, one would realize that the non-equilibrium bounce-back scheme can cause incorrect results in some cases.

In this work, a new scheme of non-equilibrium mirror-reflection for thermal boundary conditions is proposed and the properties of heat flux calculated directly from TLBM are also examined. The simulation results show that with the new scheme, TLBM can give better results both in the temperature and heat flux fields than that with the non-equilibrium bounce-back scheme.

\footnotetext{
* Corresponding author. Tel./fax: +886 223670781

E-mail address: phchen@ntu.edu.tw (P.-H. Chen).
}

\section{Lattice Boltzmann models}

TLBM uses two distributions to describe thermal systems: one for the velocity field $\left(f_{i}\right)$, the other for the temperature field $\left(T_{i}\right)$. One can refer to [5] for detail descriptions. The heat conduction is calculated by the deviations from the equilibrium $\Delta T_{i}=$ $T_{i}-T_{i}^{\mathrm{eq}}$ :

$-\alpha \frac{\partial T}{\partial \mathbf{x}}=\frac{1}{2} \sum_{i}\left(\Delta T_{i}^{\mathrm{bc}}+\Delta T_{i}^{\mathrm{pc}}\right) \mathbf{v}_{i}=\left(1-\frac{\Delta t}{2 \tau^{\prime}}\right) \sum_{i} \Delta T_{i}^{\mathrm{bc}} \mathbf{v}_{i}$

where $\tau^{\prime}$ is the relaxation time for the temperature field, the superscripts eq means equilibrium state, bc and pc denote before collisions and post-collisions, respectively. Note that $\Delta T_{i}^{\mathrm{pc}}=\left(1-\Delta t / \tau^{\prime}\right) \Delta T_{i}^{\mathrm{bc}}$ has been applied. In this study, Eq. (1) is used to calculate the temperature gradient for different boundary schemes and the D2Q9 lattice is used as illustration.

\section{Thermal boundary conditions}

Two of the most common thermal boundaries are discussed here. One is the adiabatic boundary, the other is the isothermal boundary.

\subsection{Adiabatic boundary conditions}

The adiabatic boundary conditions require no heat conduction in the normal direction. For TLBM, it means $\sum_{i}\left(\Delta T_{i} \mathbf{v}_{i}\right)_{n}=\mathbf{0}$, where the subscript $n$ denotes the normal direc- 
tion of the boundary. To meet this requirement, the non-equilibrium bounce-back scheme [3,2] imposes $\Delta T_{i}=\Delta T_{i^{\prime}}$ for the unknown distributions at boundary nodes, where the subscript $i^{\prime}$ indicates the opposite direction $\left(\mathbf{v}_{i}+\mathbf{v}_{i^{\prime}}=\mathbf{0}\right)$. There are, however, some problems for this implementation when dealing with corner nodes. Consider the lower-left corner of a rectangular domain as an example. Both $T_{6}$ and $T_{8}$ are unknown distributions. Therefore, this scheme leaves them undetermined and an additional treatment is needed. Usually the values of the equilibrium distributions are assigned [3]. Such the assignment is, in fact, arbitrary.

To avoid such the problem, we propose $\Delta T_{i}=\Delta T_{i^{*}}$, where $i^{*}$ denotes the mirror image part of the velocity $\mathbf{v}_{i}$. With this scheme, no arbitrary assignment for corner nodes is needed and the boundary temperature is determined as the same as the interior nodes. Consider the lower-left corner node again, now both $\Delta T_{6}$ and $\Delta T_{8}$ are related to $\Delta T_{7}$, which value is known from the streaming processes. We call this implementation as non-equilibrium mirror-reflection scheme because for the specular reflection by the solid surface, $\mathbf{v}_{i}$ is the corresponding outgoing direction of the incoming $\mathbf{v}_{i^{*}}$.

\subsection{Isothermal boundary conditions}

For TLBM, the isothermal boundary means $\sum_{i} \Delta T_{i}=0$. Meanwhile, constant temperatures on the boundaries also imply no temperature gradient and therefore no heat conduction along the boundaries. One scheme used by some literatures is to assign the equilibrium distributions at the boundary nodes [6,7]. This implementation would cause the discontinuity of the thermal properties and temperature jump at boundaries. To see such discontinuity, consider the deviation after collision $\Delta T_{i}^{\mathrm{pc}}=(1-\Delta t$ $\left./ \tau^{\prime}\right) \Delta T_{i}^{\mathrm{bc}}$. The equilibrium distribution scheme requires that no matter what values of $\Delta T_{i}^{\mathrm{bc}}$ are, the equilibrium distribution will be used for the streaming process, i.e. $\Delta T_{i}^{\mathrm{pc}}=0$. Therefore, it requires that $\tau^{\prime}=\Delta t$ because $\Delta T_{i}^{\mathrm{bc}} \neq 0$ in general. Since the thermal diffusivity $\alpha$ depends on $\tau^{\prime}$, it causes discontinuity of thermal properties.

Another way to meet zero sum of deviations is to impose $\Delta T_{i}=-\Delta T_{i^{\prime}}$ for unknown distributions [2]. This implementation, however, would not guarantee the heat conduction along the boundary to be zero. We categorize it as the non-equilibrium bounce-back scheme because it also involves the pair of $\mathbf{v}_{i}$ and $\mathbf{v}_{i^{\prime}}$.

To resolve the above problems, we could apply $\Delta T_{i}=-\Delta T_{i^{*}}$. Now the heat conduction along the boundary would vanish. For example, consider a lower horizontal isothermal plate. The heat conduction along the horizontal direction calculated by Eq. (1) becomes zero

$$
\left(1-\frac{\Delta t}{2 \tau^{\prime}}\right)\left[\left(\Delta T_{5}+\Delta T_{8}\right)-\left(\Delta T_{6}+\Delta T_{7}\right)\right]^{\mathrm{bc}}=0
$$

For the same reason, we call this implementation as mirror-reflection scheme.

\section{Simulation results}

Two benchmark tests are discussed. The first one is a very simple 1D transient diffusion problem. It demonstrates the assignment of equilibrium values to unknown distributions at corner nodes would affect the results. The second is a 2D Rayleigh-Bénard convection problem. It shows that the requirements on both temperature and heat flux can be satisfied if the mirror-reflection scheme is applied.

\subsection{D transient heat diffusion problem}

Consider the $1 \mathrm{D}$ transient heat diffusion problem in a rectangular box $(L \times H)$ :

$\frac{\partial T}{\partial t}=\alpha \frac{\partial^{2} T}{\partial y^{2}}$

$\mathrm{BC}: T(x, 0, t)=2, \quad T(x, H, t)=1, t>0$

$\frac{\partial T}{\partial x}(0, y, t)=\frac{\partial T}{\partial x}(L, y, t)=0$

Initially the system temperature maintains at 1 . This problem has the analytical solution and linear steady-state temperature profile. In simulations, set $L=H=20$ and then the nodes $21 \times 21\left(N_{x} \times N_{y}\right)$ are used.

Fig. 1 presents the steady-state temperature profiles next to the upper isothermal boundary for $\Delta t / \tau^{\prime}=0.75$. For $H=20$, the steadystate temperature at this location is 1.05 . The mirror-reflection schemes agree with the analytical solutions very well. Because of improper assignments at corner nodes, the non-equilibrium bounce-back scheme for adiabatic boundaries predicted nonuniform temperature profiles. This is the dominant source of simulation errors. As for the equilibrium distribution scheme for isothermal boundaries, it would produce the steady state error due to the non-uniform material properties if $\Delta t / \tau^{\prime} \neq 1$.

Let $\mathrm{Er}$ be the square root of the mean square errors compared with the analytical solutions. In general, $\mathrm{Er}$ is inversely proportional to $N_{x}^{s}$, and $s$ denotes the order of accuracy of the simulation scheme. We calculated three different lattice sizes of $21 \times 21$, $31 \times 31$, and $41 \times 41$ to determine $s$ under eight different $\Delta t / \tau^{\prime}$. Fig. 2 presents $s$ as function of $\Delta t / \tau^{\prime}$. First, note that when $\Delta t /$ $\tau^{\prime}=1$, all schemes reach the second order of accuracy. Second, for the local equilibrium scheme for isothermal boundaries, the order of accuracy is only one. Third, if the bounce-back scheme is applied for adiabatic boundaries in this benchmark case, the accuracy is decreasing with increasing $\Delta t / \tau^{\prime}$. Finally, if the mirror-reflection schemes are used for adiabatic boundaries, the order of accuracy can keep two and independent of $\tau^{\prime}$ except the local equilibrium scheme is applied for isothermal boundaries. This demonstrates that the non-equilibrium mirror-reflection scheme is better than the bounce-back one.

\subsection{D Rayleigh-Bénard convection}

The Boussinesq approximation is adopted for the 2D RayleighBénard convection with the height $H$ [8]. To make sure that the

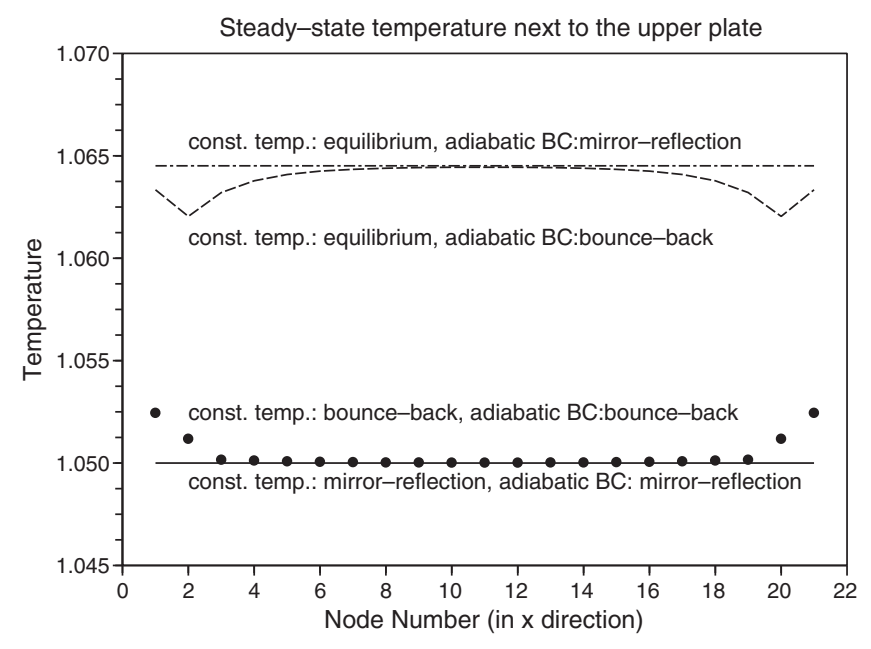

Fig. 1. Steady-state temperature profiles next to the upper isothermal boundary. 


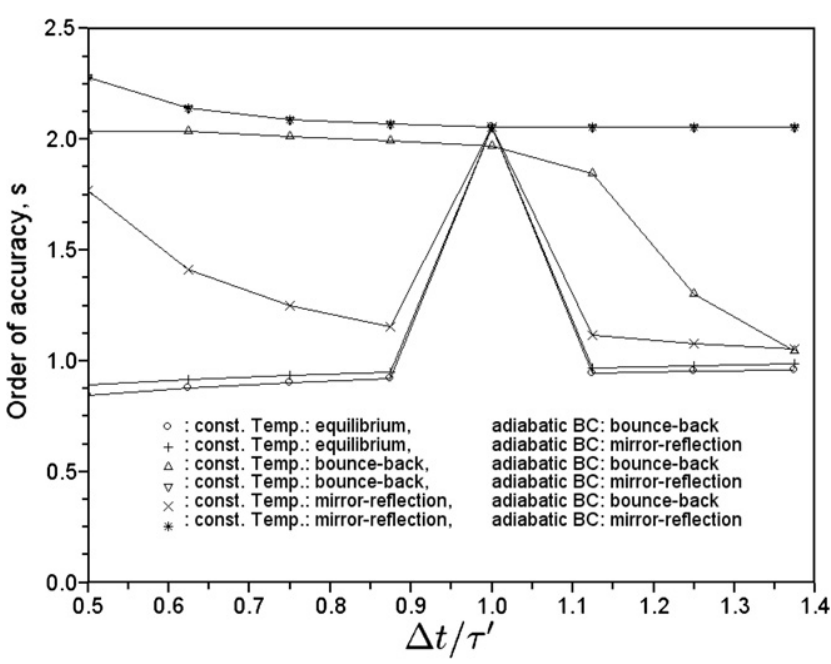

Fig. 2. Order of accuracy of different boundary schemes as function of $\Delta t / \tau^{\prime}$.

flow is in the incompressible limit, the characteristic velocity, $V^{2}=g \beta \Delta T H$, should be kept small $[3,4]$. In this study, $V^{2}=0.1$ and the Prandtl number $P r=0.71$ are applied during simulations. Thus, $v$ and $\alpha$ can be uniquely determined for each Rayleigh number
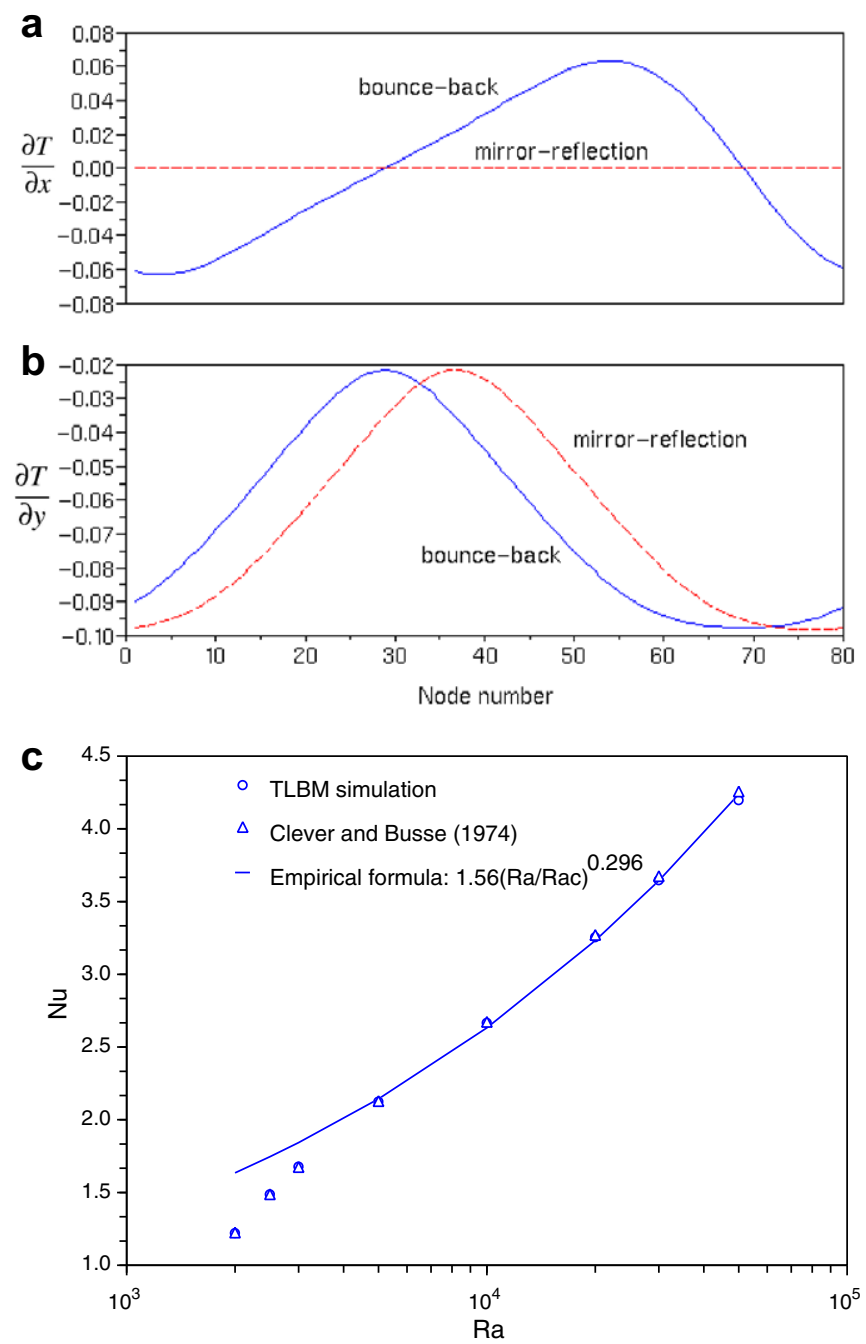

Fig. 3. Illustrations of heat conduction calculations: (a) steady-state on the lower isothermal boundary in horizontal direction $(R a=10,000)$, (b) in vertical direction, and (c) Nusselt number as function of $R a$ for $P r=0.71$.
$R a=g \beta \Delta T H^{3} / v \alpha$ once $H$ is given. As for the geometry of the lattice, since the wavelength for the lowest critical Rayleigh number, $R a_{\mathrm{c}}=1707.762$ is about 2 for nonslip boundaries [8], the aspect ratio, $L / H=2$, is set in this work. Different numbers of nodes were tested and the results were similar. The results presented here are $N_{x} \times N_{y}=80 \times 41$ for the periodic side boundary conditions. The temperatures at the lower and upper plates are set 2 and 1 , respectively. The temperature difference, $\Delta T$, is then normalized to one. The system starts from the static conductive conditions with initial small random perturbations of order $10^{-4}$ in $f_{i}$.

The temperature gradient is examined according to Eq. (1). To authors' knowledge, no literatures verified temperature gradient by this native method of TLBM. Fig. 3(a) shows the TLBM native calculations of steady-state temperature gradients in $x$ direction on the lower isothermal boundary for $R a=10^{4}$. Theoretically the temperature gradient along the isothermal surface should be zero. But the calculations by the native method of TLBM showed that if the boundary conditions are not properly imposed, the temperature gradient would not vanish even the temperatures are uniform on the boundary. One can see that from the microscopic point of view the non-equilibrium bounce-back scheme cannot meet the uniform temperature and zero temperature gradient at the same time.

Fig. 3(b) shows the steady-state temperature gradients in the vertical direction on the lower boundary. Such information can be used to determine the Nusselt number. The average Nusselt number $(\mathrm{Nu})$ is in fact the ratio of the average vertical heat flux on the boundary to the static conductive heat flux $(=k \Delta T / H)$. Fig. 3(c) presents the average Nusselt number at steady state as a function of $R a$ for $P r=0.71$. It shows good agreement with the work done by Clever and Busse [9]. This calculation should be more realistic since it resembles the experimental determination of $\mathrm{Nu}$ [10]. Besides, the computational load can be reduced significantly compared with other works [2] because no averaging over the whole domain is needed.

\section{Conclusions}

We proposed a new non-equilibrium mirror-reflection scheme of second order of accuracy for thermal boundaries in TLBM. Compared with the common non-equilibrium bounceback scheme, the non-equilibrium mirror-reflection scheme can satisfy more requirements when the predictions of heat flux are examined. This manifests the importance of heat flux as well as temperature to evaluate numerical models for thermal systems. The present work is also the first one to verify the temperature gradient by the native method of TLBM. In addition, We demonstrated an efficient and realistic way to determine the Nusselt number for RB convection by calculating the heat flux on the boundaries. Not only does it resemble the real physical measurements, it can reduce significantly the computational load because only boundary nodes are involved instead of averaging over the whole domain.

\section{Acknowledgements}

The authors thank the financial support of the National Science Council of Taiwan, Republic of China under Contract No. NSC962628-E-002-197-MY3.

\section{References}

[1] X.W. Shan, Simulation of Rayleigh-Bénard convection using a lattice Boltzmann method, Phys. Rev. E 55 (1997) 2780-2788.

[2] X. He, S. Chen, G.D. Doolen, A novel thermal model for the lattice Boltzmann method in incompressible limit, J. Comput. Phys. 146 (1998) $282-300$. 
[3] Y. Peng, C. Shu, Y.T. Chew, Simplified thermal lattice Boltzmann model for incompressible thermal flows, Phys. Rev. E 68 (2003) 026701-1-026701-8.

[4] X.Y. He, Q.S. Zou, L.S. Luo, M. Dembo, Analytic solutions of simple flows and analysis of nonslip boundary conditions for the lattice Boltzmann BGK model, J. Stat. Phys. 87 (1997) 115-136.

[5] G. Barrios, R. Rechtman, J. Rojas, R. Tovar, The lattice Boltzmann equation for natural convection in a two-dimensional cavity with a partially heated wall, J. Fluid Mech. 522 (2005) 91-100.

[6] G.H. Tang, W.Q. Tao, Y.L. He, Simulation of fluid flow and heat transfer in a plane channel using the lattice Boltzmann method, Int. J. Mod. Phys. B 17 (2003) 183-187.
[7] P.H. Kao, T.F. Ren, R.J. Yang, An investigation into fixed-bed micro-reactors using lattice Boltzmann method simulations, Int. J. Heat Mass Transfer 50 (2007) 4243-4255.

[8] S. Chandrasekhar, Hydrodynamic and Hydromagnetic Stability, Dover, New York, 1981.

[9] R.M. Clever, F.H. Busse, Transition to time-dependent convection, J. Fluid Mech 65 (1974) 625-645.

[10] P. Kolodner, R.W. Walden, A. Passner, C.M. Surko, Rayleigh-Bénard convection in an intermediate-aspect-ratio rectangular container, J. Fluid Mech. 163 (1986) 195-226. 